\title{
Photonic Quantum Simulators
}

\section{Citation}

Aspuru-Guzik, Alan, and Philip Walther. 2012. "Photonic Quantum Simulators." Nature Physics 8 (4) (April 2): 285-291. doi:10.1038/nphys2253. http://dx.doi.org/10.1038/nphys2253.

\section{Published Version}

doi:10.1038/nphys2253

\section{Permanent link}

http://nrs.harvard.edu/urn-3:HUL.InstRepos:11881655

\section{Terms of Use}

This article was downloaded from Harvard University's DASH repository, and is made available under the terms and conditions applicable to Other Posted Material, as set forth at http:// nrs.harvard.edu/urn-3:HUL.InstRepos:dash.current.terms-of-use\#LAA

\section{Share Your Story}

The Harvard community has made this article openly available.

Please share how this access benefits you. Submit a story.

Accessibility 


\title{
Photonic Quantum Orreries:
}

\section{Review of photonic quantum simulations.}

\author{
Alán Aspuru-Guzik ${ }^{1}$ and Philip Walther ${ }^{2}$ \\ ${ }^{1}$ Department of Chemistry and Chemical Biology, Harvard University, Cambridge, \\ Massachusetts 02138, USA, \\ ${ }^{2}$ Faculty of Physics, University of Vienna, Boltzmanngasse 5, Vienna A-1090, Austria.
}

Quantum simulators are controllable quantum systems that can be used to mimic other quantum systems and are thus being able to tackle problems that are intractable on conventional computers. Today's available photonic quantum technology is reaching the stage where significant advantages arise for the simulation of particular interesting questions in quantum chemistry, quantum biology and solid-state physics. We review recent progress in the field of photonic quantum simulation, which should break the ground towards the realization of versatile quantum simulators. In addition, photonic quantum systems offer also the unique benefit of being mobile over free space and in waveguide structures, which opens new perspectives to the field by enabling the natural investigation of quantum transport phenomena.

About two thousand years ago, the Greeks built orreries, mechanical devices constructed to simulate the classical dynamics of planetary motion. The construction of orreries was possible due to the technological advances in mechanics and materials science of the early times. One of the current directions in quantum science is the 
development of modern quantum orreries: quantum mechanical simulators of chemical and physical processes at the scale where quantum effects are crucial.

It was Feynman who proposed the radical idea for the efficient simulation of quantum systems. One could employ a controllable quantum system to reproduce the dynamics and the quantum state of the original system of study. Classical computers are unable to simulate quantum systems efficiently, because they need to enumerate quantum states one at a time. Quantum simulators allow one to bypass the exponential barriers that are imposed by entanglement and the superposition principle of quantum mechanics, which inhibit classical computers from making progress in calculation time. Thirty years after Feynman's original proposal ${ }^{1}$, quantum simulators of physical systems are being successfully constructed with a variety of quantum architectures, such as atoms ${ }^{2-5}$ trapped ions ${ }^{6-11}$, NMR ${ }^{12,13}$, superconducting circuits ${ }^{14}$ as well as single photons ${ }^{15-22}$, which are the focus of this review. However, even though there are many recent exciting developments in several quantum architectures, such as ion-trap quantum computing ${ }^{8,10}$, no physical implementation seems to have a definite edge in all aspects of the race at this point.

\section{Quantum simulation strategies}

With respect to level of detail, there are two types of quantum simulators. For the first type, the goal is simulating a collective property such as a quantum phase transition, and for this, global or coarse-grained control of the quantum particles is usually enough to observe these phenomena. The second class of simulators requires precise local control and addressability of individual particles to provide a platform for understanding mesoscopic and molecular systems. 
Simulators can also further be classified into digital when using discrete quantum gate operations and analog (including adiabatic models) when implementing a surrogate Hamiltonian in an analog fashion ${ }^{23}$. However, there is also the possibility of constructing hybrid systems that combine quantum gate models and analog quantum simulation techniques ${ }^{20}$.

\section{Photonic quantum technology}

Each technology has its strengths, and here we discuss the inherent advantages of photonic technology for precise single-particle quantum control and tunable measurement-induced interactions to realize local photonic quantum simulators. One of the salient features of photons is that they do not interact easily. This results in a naturally decoherence-free system but also complicates the generation of entanglement. Photons can be easily manipulated and individually addressed with high precision by employing simple components at room temperature. These features provide the second advantage of photonic simulators: Photons are easily moved either in free space or waveguides and thus are not restricted to nearest neighbors. The mobility of photons, ideally on a single chip, will allow almost arbitrary interconnections and facilitate the simulation of complex and non-local many-body interactions.

On the challenge side, photonic simulators could be potentially scalable, if we find a technology for the controlled generation of single photons. This is an area of fertile research and an overview of the photonic quantum toolbox is given in box 2 .

As shown in the examples below, photons are a great platform for simulating quantum phenomena of small-sized quantum systems. The photons' mobility enables even single-photon experiments to simulate quantum walks ${ }^{18,19,21,22}$ and topological phases ${ }^{24}$. 
Recently, Lanyon et al. ${ }^{17}$ were able to simulate the hydrogen molecule by using two entangled photons. One entangled photon represented the wave function of a two-level system that represents two spin-orbitals, and the other was used to read out the molecular energy. But the story does not end with a two photonic qubits. Armed with four photons, Ma et al. ${ }^{20}$ have simulated frustrated valence-bond states by achieving quantum control over four particles. The tunable interaction between two entangled photon pairs allowed studying the distribution of pairwise quantum correlations as a function of the competing spin-spin interactions.

In the following sections, we will elaborate further on these examples, and describe other recent and ongoing applications of quantum simulation using photons. The list is by no means exhaustive and is meant to be representative of the current state-of-the-art.

\section{Chemistry and Biology}

Quantum chemistry and band-structure calculations account for up to $30 \%$ the supercomputer time employed at supercomputer centers ${ }^{25}$. The most employed techniques include density functional theory and tractable correlated electronic structure methods ${ }^{26}$. Although these methods can be used to predict novel materials ${ }^{27}$, they are approximate in nature. Formally, the exact solution of the Schrödinger equation within a given numerical basis scales exponentially with the number of basis functions. This is known as the curse of dimensionality and is one of the two fundamental reasons that quantum chemistry is hard on a quantum computer. The second reason is that the classical computer might not be able to converge to the quantum state of all possible molecular input states. In computer science, one usually deals with the worst possible 
instance, which might correspond to a very strongly correlated molecule or material. Nevertheless, we conjecture ${ }^{28,29}$ that it will be easy to prepare typical instances of molecules on quantum computers. Recently, Schuch and Verstraete showed that finding an exact density functional for interacting electrons, would be Quantum Merlin Arthur (QMA)-Hard, that is, belonging to a class of problems thought too hard even for quantum computers ${ }^{30,31}$.

In 2005 , a quantum chemistry algorithm was introduced ${ }^{28}$ that is linear scaling in the number of qubits and fifth-order scaling in terms of the number of quantum gates ${ }^{32}$. This algorithm is based on the original proposal by Abrams and Lloyd ${ }^{33,34}$ and uses quantum phase estimation to obtain molecular eigenvalues. These algorithms usually rely on time slicing via the Trotter formula, which results in a large number of gates as a function of system size.

The simplest possible quantum circuit for quantum chemistry on a quantum information processor has already been implemented. The hydrogen molecule Hamiltonian in the smallest atom-centered chemistry basis results in a $6 \times 6$ matrix that has two $2 \times 2$ blocks and two $1 \mathrm{x} 1$ blocks. The $2 \times 2$ blocks can be diagonalized by carrying out the iterative phase estimation algorithm ${ }^{35}$. Lanyon et al. ${ }^{17}$ performed the experiment using two entangled photons and taking advantage of additional photonic degrees of freedom to implement an arbitrary controlled-unitary evolution ${ }^{36}$ (Figure 1). A few months later, the experiment was also realized using an NMR quantum system ${ }^{13}$. Future experiments will require a scalable photonic architecture, as the number of CNOT gates required scales as the fifth power of the system size.

However, in the case of photonic quantum systems, arbitrary unitary matrices can be also implemented by interferometric beamsplitter arrays or so-called multi-port arrays 
${ }^{37}$. A recent achievement, micro-optics using integrated waveguides ${ }^{21,22,38-40}$, opens up promising perspectives on the scale up of future quantum simulation experiments.

\section{Simulation of quantum walks and tight-binding Hamiltonians}

It was recognized several years ago ${ }^{41}$ that waveguide arrays could be employed to simulate quantum walks ${ }^{42}$ and, if decoherence is involved, quantum stochastic walks ${ }^{43}$. A walk is just a distribution evolving over a given graph following a defined equation of motion. Classical walks involve classical probability distributions that follow a classical transition matrix. Quantum walks involve a distribution of amplitudes following Schrödinger dynamics. Finally, quantum stochastic walks involve density matrices following open quantum system dynamics. Walks can be formalized in both discretetime (DT) and continuous time (CT). Although originally devised for quantum computation, quantum walks can be used to simulated tight-binding Hamiltonians, either as closed systems, or under decoherence.

Quantum walks have been realized using bulk optics ${ }^{24,44-46}$, and waveguides ${ }^{19,22,40,41,47}$. Single-particle quantum walks can also be carried out with coherent classical light, but truly novel effects happen when more than one photon is employed ${ }^{21,22,40}$. The use of loop-based architectures has led to realizations of up to 28 steps in a discrete walk ${ }^{45}$.

Excitation transfer in biological systems - Recent ultrafast dynamical experiments have shown the existence of long-lived quantum coherent oscillations in biological systems ${ }^{48-53}$. These long-lived oscillations are due to a convergence of timescales of the biological system that results in quantum oscillations that last up to picoseconds at room temperature. The open quantum system (electrons in a bath of phonons) is a target for 
quantum simulation. Many connections with quantum information have resulted in the description of this process as an environment-assisted quantum walk ${ }^{51}$, environmentassisted quantum transport (ENAQT) ${ }^{52,53}$ or a quantum stochastic walk ${ }^{54}$. In these different theoretical frameworks, the interplay of the bath and the environment at the natural parameters is the key to the efficiency of the photosynthetic complex.

The first quantum optics experiment along this line simulated a quantum stochastic walk (QSW) that interpolates between the quantum and the classical walk using a single-photon. This was realized using spatial degrees of freedom and calcite beam displacers ${ }^{44}$. Up to six steps of the discrete-time quantum walk were performed using this approach. A similar experiment was later realized in a loop configuration ${ }^{46}$.

Another recently-proposed approach to simulate the transition from coherent to incoherent transport, as well as ENAQT involves the use of a set of coupled optical cavities transversed by a single photon ${ }^{55}$.

A recent experiment ${ }^{40}$ showcased quantum correlations between two photons in threedimensional directly-written waveguide arrays. This experiment could be a precursor for the simulation of photosynthetic complexes, where a delocalization over different bacteriochlorophyll molecules is very important to describe the correct energy transfer dynamics.

\section{Condensed Matter Physics}

Valence Bond States. In addition to the superior level of quantum control, photonic quantum simulators allow the use of quantum interference at beamsplitters which can lead to interesting photon-entanglement that corresponds to ground states of correlated chemical or solid-state systems ${ }^{56}$. Ma et al. ${ }^{20}$ have shown that frustration in 
Heisenberg-interacting spin systems can be investigated by such a photonic quantum simulator. The pairing of quantum correlations of spin systems is an important mechanism in chemical or so-called valence bonds, where two electrons from different atoms share an anti-correlated spin state due to the Pauli principle. Valence-bond states are of particular interest because it was conjectured that a transition from a localized valence-bond configuration to the superposition of different valence-bond states might explain high-temperature superconductivity in cuprates ${ }^{57}$.

The same quantum correlation of valence-bond states can be simulated by a pair of photons that is maximally entangled in polarization, i.e. that the two photons are always orthogonal polarized. Ma's experiment used two entangled photon pairs in a singlet state to simulate the spin of a Heisenberg-interacting spin tetramer, where the singlet state is analogous to the anti-ferromagnetic coupling of two spin-1/2 particles or valence-bond state. Then an analog quantum simulation was performed by superimposing photons from each pair at a beamsplitter with a tunable splitting ratio, followed by a measurement of the photons in the output ports (Figure 2a). Depending on the interaction strength, the transition from local to resonant valence bonds ground state could be observed. The precise addressability of individual photons provided insight into the pairwise quantum correlations and allowed to observe that the energy distributions are restricted by the role of quantum monogamy ${ }^{58,59}$. Such quantum simulation experiments will be of interest for quantum chemistry with small numbers of particles and might allow in the near future the simulation of aromatic systems such as benzene (Figure 2b). 
Topological quantum states. A recent area of interest in quantum physics is the study of quantum phases with special topological properties that could yield topologicallyprotected states. These states, in turn, could be employed for quantum information processing. Condensed-matter systems such as topological insulators have received a lot of theoretical and experimental study.

The direct observation of topological states is a challenging experimental problem, so one approach to the problem is that of building an orrery where the effect can be observed. Recently ${ }^{24}$, an optical setup similar to that employed for the simulation of quantum walks ${ }^{44}$ was modified to achieve a one-dimensional topologically protected pair of states, using a periodic-driving Hamiltonian. This resulted in the production of a direct experimental observation of these states using a discrete-time quantum walk (Figure 3).

\section{Particle Statistics and Elementary Interactions}

In quantum physics two fundamental particle classes exist: bosons that obey BoseEinstein statistics and fermions that obey Fermi-Dirac statistics. While bosonic particles can occupy the same quantum state and bunch, fermionic particles must follow the Pauli-exclusion principle and thus anti-bunch. These non-classical particle statistics can be simulated with photons that are experiencing multi-port beamsplitter structures in bulk or integrated optics ${ }^{60}$. Since the early days of correlated photon-pair generation, photon bunching is observed when superimposing indistinguishable single photons at beamsplitters ${ }^{61}$. However, the situation changes when polarized photons are used as input, because of either sharing a symmetric (triplet) or an anti-symmetric (singlet) state that leads to respectively boson-like bunching or fermionic-like antibunching. Although 
the ability to observe non-bosonic statistics with photons was originally used in quantum information experiments ${ }^{60,62,63}$, recent demonstrations of two-photon interference in integrated waveguides enabled the simulation of quantum interference of fermions, bosons and anyons ${ }^{21,22}$.

In general the flexibility of photons makes such systems promising for studying a variety of different quantum physical properties. For example, the theoretical work by Semião and Paternostro ${ }^{64}$ suggests to use photons for obtaining insights also into particle physics. The authors propose to use a combination of CNOT gates and multiports ${ }^{37}$ to emulating the nucleonic spin states that result from the combination of their quark components. Obviously, such proposed quantum simulation experiments may rather cover the first quark models than the full picture using the theoretical framework of quantum chromodynamics. But, in analogy orraries, such experiments might provide new insights into phenomenological properties of nucleonic states. Nevertheless, the fact that state-of-the art technology enables the mimicking of such three- and more-body interactions underline the applicability of photonic networks for future simulation experiments of fundamental phenomena that exist in subatomic particles.

\section{Perspective and Outlook}

The holy grail for future experiments will be the outperformance of existing conventional supercomputers for the task of simulating quantum systems. Although the resource requirements are less demanding in the case of quantum simulators, the necessary number of input states and the measurement-based processing make such benchmark experiments are currently out of reach. However, it was recently shown that the simulation of bosonic particle statistics might be the first application in which 
photons will outperform classical devices. The remarkable work by Aaronson and Arkhipov ${ }^{31}$ strongly suggests that rudimentary photonic networks built entirely out of linear-optical elements cannot be efficiently simulated by classical computers. This has two immediate consequences: Firstly, that already the bosonic nature of photons itself is hard to simulate on conventional computers, which means that the particle symmetry alone leads to fundamental complexity that goes beyond what is expected of classical computers. And secondly, that it is likely that the first benchmark quantum simulation experiment might be built purely from passive linear-optical elements without the necessity of additional ancilla photons for introducing measurement-based interactions. In other words, sending identical photons through an optical network without any kind of adaptive measurements might be the first simulation of complex phenomena that are classically intractable under plausible assumptions.

If progress on future quantum orreries based on the many available quantum technologies is steady, we expect that grand computational challenges such as the accurate simulation of molecules and materials will become accessible. En route to realizing this long term goal, photonic quantum simulators provide a useful test bed for the realization of Feynman's dream and have already broken ground with the host of few-photon quantum orreries discussed here.

\section{Acknowledgements}

We thank Ivan Kassal for his careful comments on the manuscript. Max Tillman, Matthias Tomandl and Lauren Kaye prepared graphics for the manuscript. A.A.-G. also acknowledges support from the National Science Foundation under the Center for Chemical Innovation (CCI) program, as well as the Camille and Henry Dreyfus and 
Sloan Foundations for support. Ph.W. acknowledges support from the European Commission, QESSENCE (No 248095), the JTF, the Austrian Science Fund (FWF), [SFB-FOCUS] and [Y585-N20], and from the Air Force Office of Scientific Research, Air Force Material Command, USAF, under grant number FA8655-11-1-3004. 


\section{Box 1: What is a quantum orrery?}

A quantum orrery or simulator is a quantum device capable of reproducing the behavior of another quantum system. Although quantum computers have shown to be able to universally simulate quantum systems, they are often not necessary. Unlike quantum computers, quantum simulators can have more limited control and measurement possibilities. Many types of simulators can be foreseen: Simulators can either reproduce the time dynamics of a given system, or the can be used to recreate quantum states of interest.

In a quantum computer simulation, a quantum is mapped to a mathematical model that represents it. For example, a molecule can be mapped to the non-relativistic Schrodinger equation. This equation can be then executed by a quantum software layer, which in turn is mapped to quantum hardware. The simulator is employed to measure a desired property, in this example, the molecular energy. Not all desired properties are readily available in polynomial time: A full map of the wave function of the system would require an exponentially-scaling number of measurements. Therefore, for a successful quantum orrery experiment, the stages of preparation, simulation and measurement have to be designed such that they are carried out in a quantum system in a computationally efficient manner (Box Figure 1). 


\section{Box 2: The photonic toolbox: today and tomorrow}

Photonic quantum systems ${ }^{65}$ are among the most mature and promising approaches for the realization of quantum computers and quantum simulators. Single photons are excellent carriers of quantum information due to their robustness and their mobility which literally enables them to transmit information at the speed of light. Quantum-bits, which represent any physical two-level quantum system, are often encoded in the polarization state of photons, because of being easily manipulated with high precision by using birefringent phase retarders. In addition, photons also provide other degrees of freedom such as path or angular momentum for encoding quantum information.

The downside is that photons barely interact, which puts the main challenge on the engineering of photon-photon interactions, which are crucial for the two-qubit gate operations or the preparation of multi-photon entanglement using single photons as input states. In 2001, the seminal work of Knill, Laflamme and Milburn ${ }^{66}$ has shown that effective nonlinearites can be introduced via the measurement process and that scalable photonic quantum computing is possible by using only linear optical circuits, single-photon sources, and detectors. It was shown that the introduction of ancillary photons do not only allow to herald successful gate operations ${ }^{67}$ but also to constructed protocols in which probabilistic two-photon gates are teleported into a quantum circuit with high probability. This opened a legitimate path to build large-scale quantum simulators that in contrast to many other physical architectures do not face the technical challenges of low temperature and vacuum conditions.

Therefore the controlled generation of single photons is at present the main hurdle for the scale-up of photonic quantum simulators. The requirements to achieve quantum interference between photons emitted from independent sources, made multi-photon 
experiments typically relying on the process of parametric down-conversion ${ }^{68}$, where indistinguishable pairs of photons are generated by a strong laser pulse in a nonlinear crystal. Unfortunately this process is spontaneous and thus creates photon-pairs at random times, which restricts the scalability, especially when dealing with the emission of multiple photon-pairs and standard detectors without photon-number resolution. Other leading technologies in this effort are based on different physical systems including single trapped atoms and atomic ensembles, quantum dots, or nitrogenvacancy centers in diamond ${ }^{65}$. Once the technical challenges of low outcoupling efficiencies, the uncertainty in emission time and the distinguishability in frequency of the created photons are addressed, these systems will become very promising candidates for controlable single-photon sources that can be integrated on chips. Similarly, significant effort is put in high-efficient superconducting detection units that can distinguish the number of detected photons ${ }^{69-75}$ such that errors due to additional photons can be excluded for improving quantum state and quantum gate fidelities. Therefore, the ultimate vision of combining multi-photon sources, circuits and detection units on a single chip, seems to be a very challenging long lasting goal, but is certainly not out of reach (Box Figure 2). 


\section{Box 3: Towards outperforming classical computers}

A question commonly posed to researchers in quantum information is when a quantum computer or quantum simulator is expected to outperform a classical computer. Classical computers have a head start of many years. The first classical simulations were performed in the MANIAC computer at Los Alamos National Laboratories in the 1940's. The first photonic quantum simulations are just being realized this decade, almost 70 years later. The current computational power has enabled approximate calculations such as the full molecular dynamics simulation of a protein ${ }^{76}$. These cannot be directly compared with the few-qubit experiments carried out nowadays. The promise of quantum simulation is to provide exact simulations with a polynomiallyscaling in quantum resources. This would be crucial to benchmark currently approximate methods. Nevertheless, estimates have been made of where the crossover could occur. For quantum chemistry simulation, a quantum simulator would beat the best algorithms on a classical computer beyond approximately 150 quantum bits. Quantum simulators could have a lower crossover threshold for strongly correlated systems, as these are intractable using classical computers (Box Figure 3). 


\section{Box-Figure 1:}

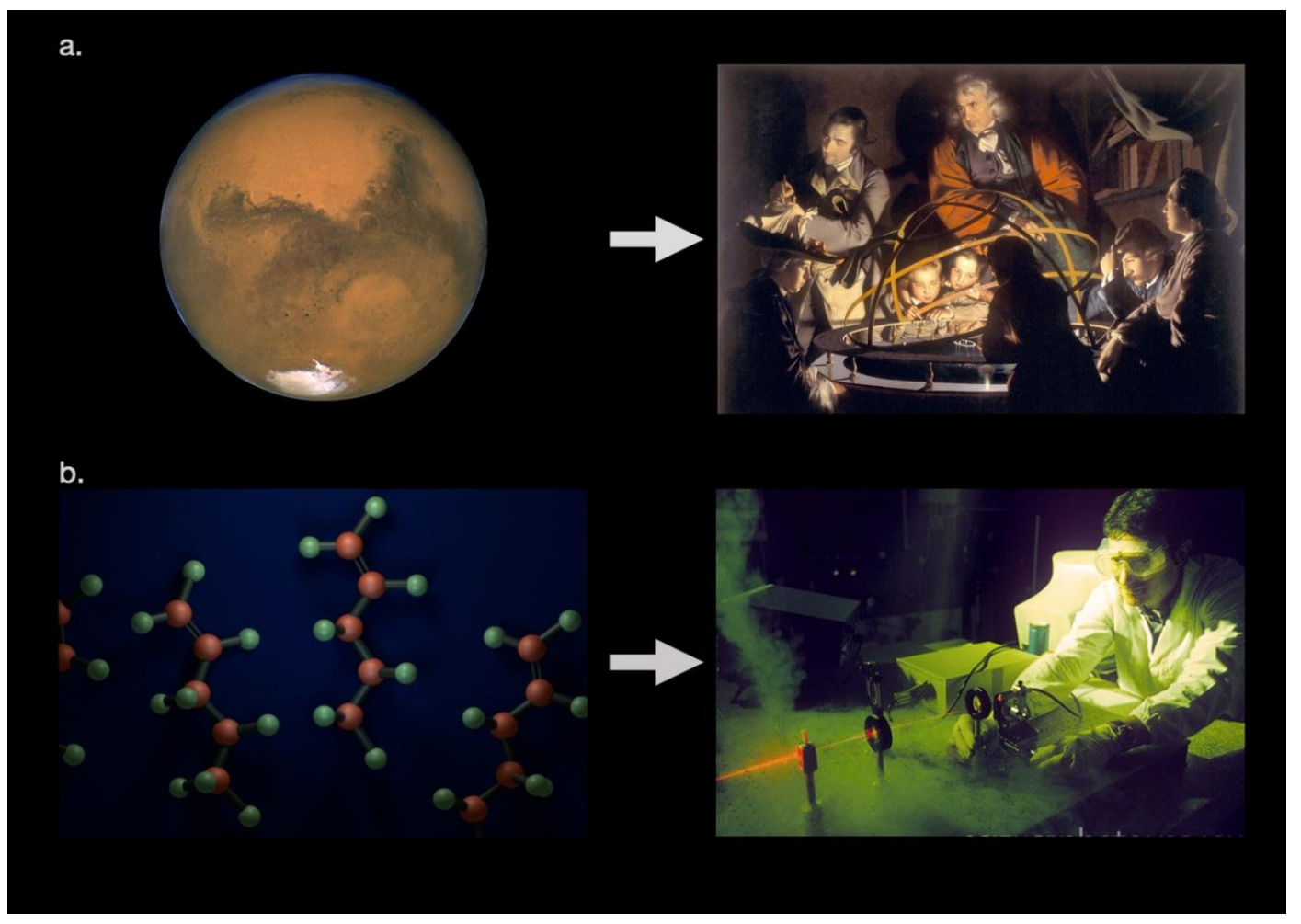

Box Figure 1 (included in box): Mechanical technology allowed for the development of classical orreries: The movement of the planets was simulated using the technology of the day (gears). Quantum technology offers the opportunity of simulating systems of scientific and technical relevance by mapping their dynamics to those of controllable quantum devices, such as photonic quantum optics. 


\section{Box-Figure 2:}

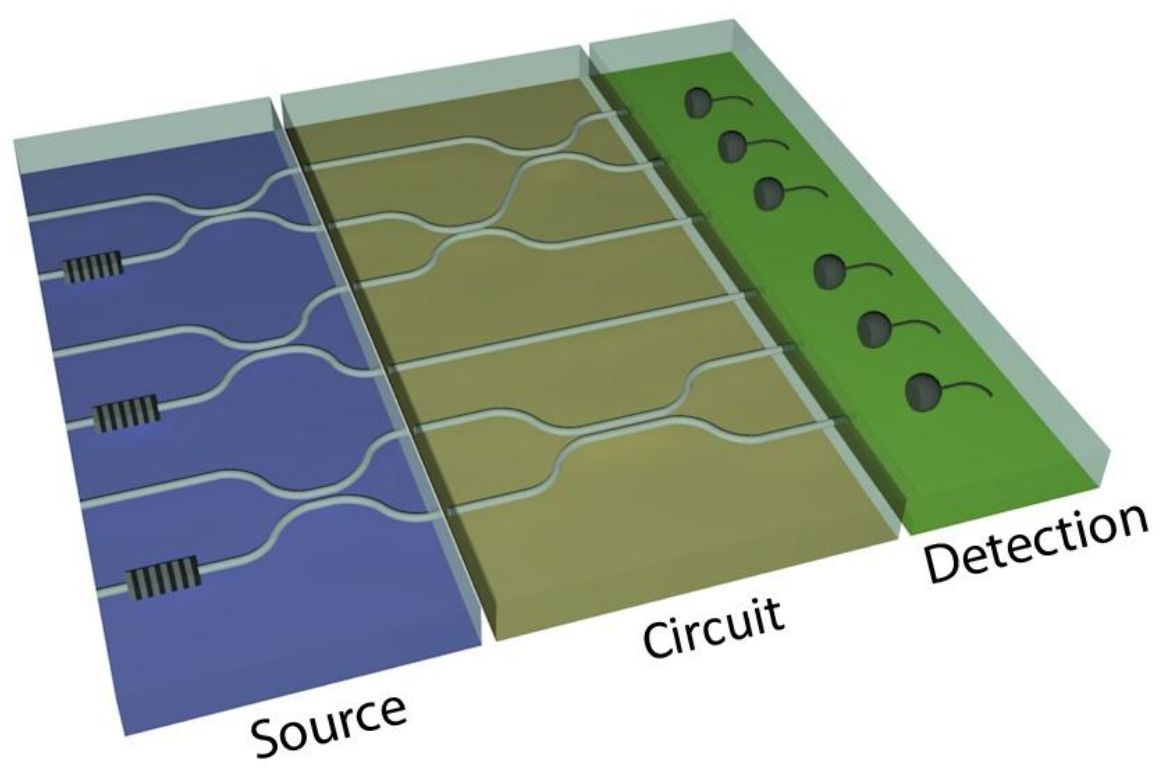

Box Figure 2 (included in box): Vision of an integrated photonic quantum simulator.

The vast development of micro-optics and waveguide technologies open up promising perspectives to integrate single-photon sources, tunable circuits and high-efficiency detection units on one single chip. 


\section{Box-Figure 3:}

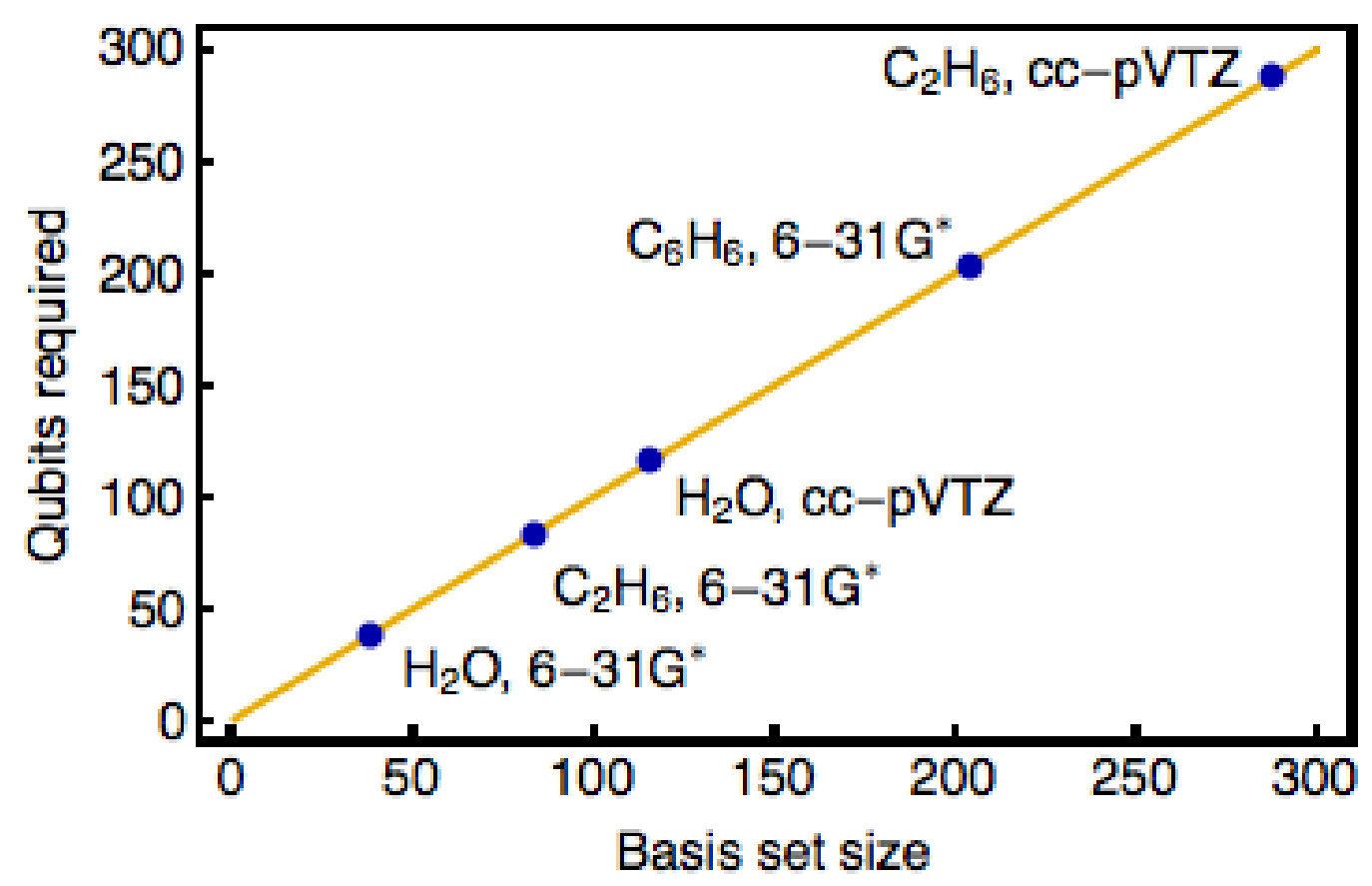

Box Figure 3 (included in box): Number of quantum bits required for molecular electronic structure calculations. Several molecules are shown, using different quantum chemistry basis sets ${ }^{29,77}$. A Full Configuration Interaction (FCI) on 75 basis functions is currently intractable for classical computers. A quantum simulator with approximately 150 logical quantum bits would be able to outperform quantum computers at tasks of quantum chemistry such as the simulation of chemical reaction dynamics and molecular electronic structure. 


\section{Figure 1:}

(a)

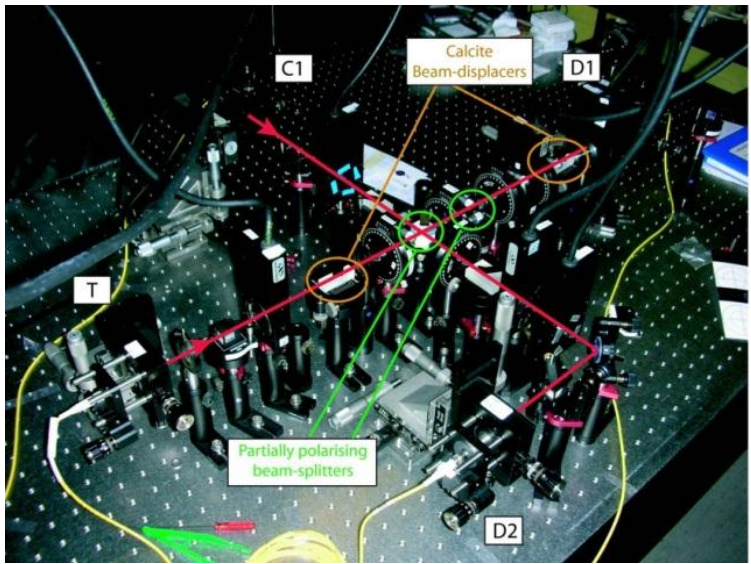

(b)

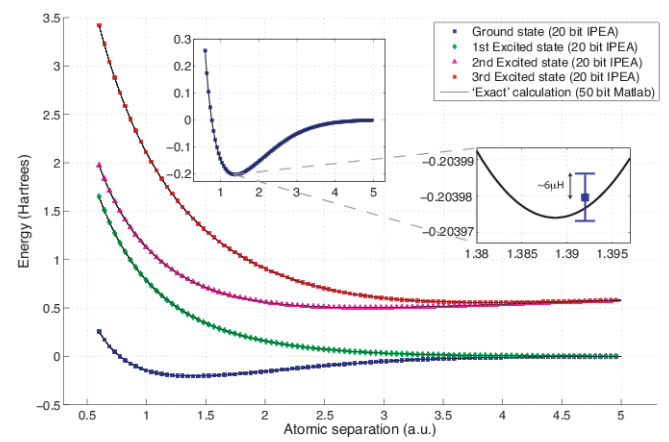

Figure 1: First quantum chemistry experiment on a quantum information processor. (a) Quantum optics experiment for simulating the energy of the hydrogen molecule in the minimal basis set. A pair of entangled photons implements an iterative phase estimation scheme where one of the photons represents two $2 \times 2$ blocks of the $6 \times 6$ full configuration interaction matrix of $\mathrm{H}_{2}$ in the minimal quantum chemistry basis set ${ }^{17}$. (b) Plot of the molecular energies of the different electronic states as a function of interatomic distance obtained with the device to 20 bits of precision by means of an iterative phase estimation procedure and majority voting. 


\section{Figure 2:}

(a)

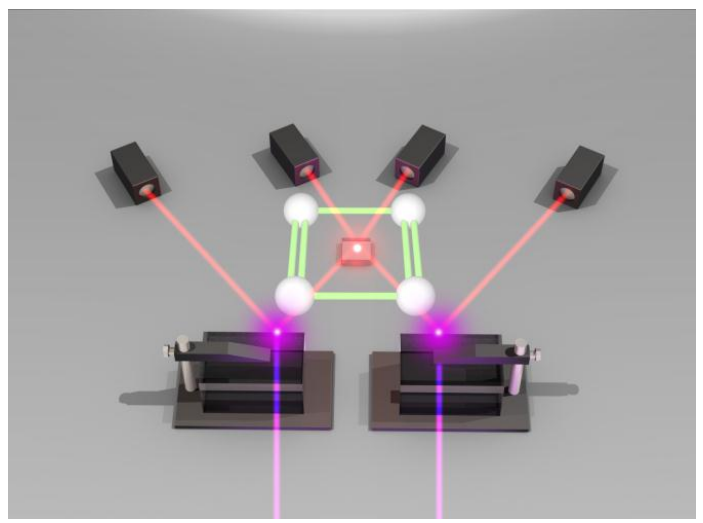

(b)

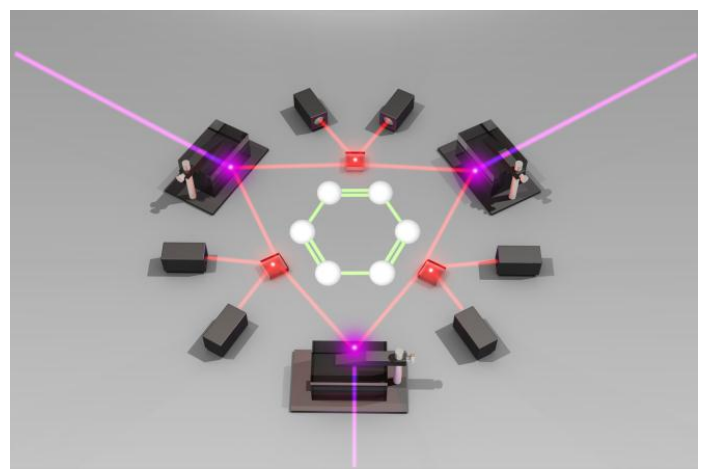

Figure 2: Artistic view of the photonic quantum simulation of delocalized chemical bonds. (a) Two entangled photon pairs are generated via the process of parametric down-conversion. Superimposing one single photon from each pair at a tunable beamsplitters results in quantum interference such that the measured four-photon coincidences correspond to the ground state, for example of a Heisenberg-interacting spin tetramer. Dependent on the beamsplitter reflectivity frustration in valence bond states or so-called spin liquid states could be investigated 20 (b) Future experiments using more entangled photon pairs may allow the study of ground state properties of molecular ground states, such as the delocalized bonds in benzene. 


\section{Figure 3:}

(a)

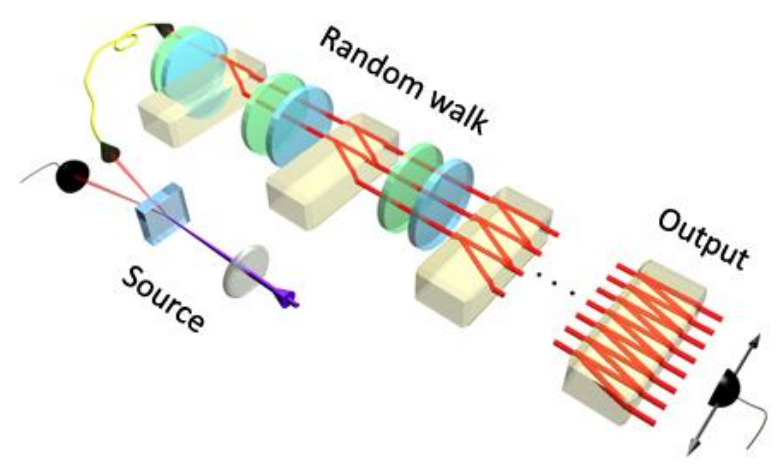

(b)

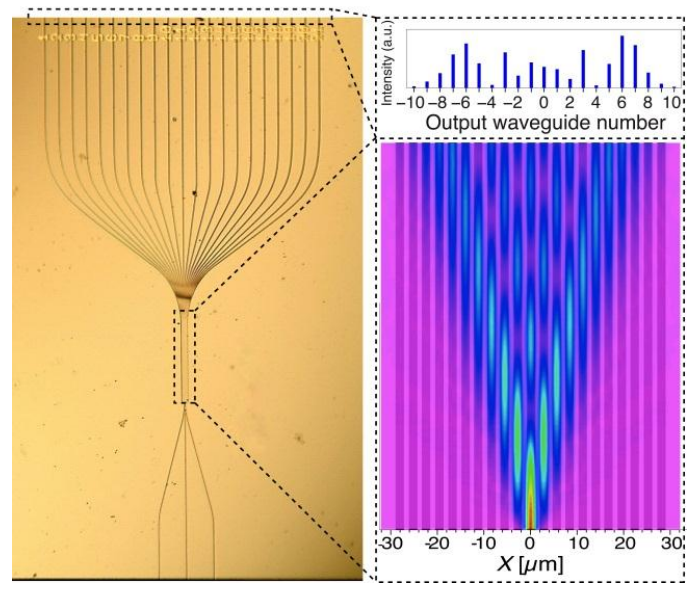

Figure 3: Photonic quantum circuits are well suited for the simulation of quantum and quantum stochastic walks. (a) The bulk optics setup was employed to simulate a quantum stochastic walk transition between a pure quantum walk and a classical walk ${ }^{44}$. (b) Continuously coupled waveguide arrays were also used for realizing correlated photon quantum walks ${ }^{19}$. An optical micrograph of a 21-waveguide array showing the three input waveguides bending into the $700-\mu \mathrm{m}$-long coupling region, before being detected at the output ports. The output pattern (small inset) and a simulation of the intensity of laser light propagating in the array (bigger inset) are also shown. 


\section{Bibliography}

1. Feynman, R. Simulating Physics with Computers. International Journal of Theoretical Physics 21, 467-488 (1982).

2. Bakr, W.S., Gillen, J.I., Peng, A., Folling, S. \& Greiner, M. A quantum gas microscope for detecting single atoms in a Hubbard-regime optical lattice. Nature 462, 74-77 (2009).

3. Trotzky, S. et al. Suppression of the critical temperature for superfluidity near the Mott transition. Nat Phys 6, 996-1004 (2010).

4. Weitenberg, C. et al. Single-spin addressing in an atomic Mott insulator. Nature 471, 319-324 (2011).

5. Lewenstein, M. et al. Ultracold atomic gases in optical lattices: mimicking condensed matter physics and beyond. Advances in Physics 56, 243-379 (2007).

6. Friedenauer, A., Schmitz, H., Glueckert, J.T., Porras, D. \& Schaetz, T. Simulating a quantum magnet with trapped ions. Nat Phys 4, 757-761 (2008).

7. Gerritsma, R. et al. Quantum simulation of the Dirac equation. Nature 463, 6871 (2010).

8. Barreiro, J.T. et al. An open-system quantum simulator with trapped ions. Nature 470, 486-491 (2011).

9. Islam, R. et al. Onset of a Quantum Phase Transition with a Trapped Ion Quantum Simulator. Nature Communications 2, 377 (2011).

10. Lanyon, B.P. et al. Universal Digital Quantum Simulation with Trapped Ions. Science 334, 57 -61 (2011).

11. Kim, K. et al. Quantum simulation of frustrated Ising spins with trapped ions. Nature 465, 590-593 (2010).

12. Peng, X., Zhang, J., Du, J. \& Suter, D. Quantum Simulation of a System with Competing Two- and Three-Body Interactions. Phys. Rev. Lett. 103, 140501 (2009).

13. Du, J. et al. NMR Implementation of a Molecular Hydrogen Quantum Simulation with Adiabatic State Preparation. Phys. Rev. Lett. 104, 030502 (2010).

14. Neeley, M. et al. Emulation of a Quantum Spin with a Superconducting Phase Qudit. Science 325, 722-725 (2009).

15. Lu, C.-Y. et al. Demonstrating Anyonic Fractional Statistics with a Six-Qubit Quantum Simulator. Phys. Rev. Lett. 102, 030502 (2009). 
16. Pachos, J.K. et al. Revealing anyonic features in a toric code quantum simulation. New J. Phys. 11, 083010 (2009).

17. Lanyon, B.P. \& others Towards quantum chemistry on a quantum computer. Nat Chem 2, 106-111 (2010).

18. Broome, M.A. et al. Discrete Single-Photon Quantum Walks with Tunable Decoherence. Phys. Rev. Lett. 104, 153602 (2010).

19. Peruzzo, A. et al. Quantum Walks of Correlated Photons. Science 329, 15001503 (2010).

20. Ma, X.-song, Dakic, B., Naylor, W., Zeilinger, A. \& Walther, P. Quantum simulation of the wavefunction to probe frustrated Heisenberg spin systems. Nat Phys 7, 399-405 (2011).

21. Matthews, J.C.F. et al. Simulating quantum statistics with entangled photons: a continuous transition from bosons to fermions. arXiv:quant-ph/1106.1166 (2011).

22. Sansoni, L. et al. Two-particle bosonic-fermionic quantum walk via 3D integrated photonics. arXiv:quant-ph/1106.5713 (2011).

23. Buluta, I. \& Nori, F. Quantum Simulators. Science 326, 108-111 (2009).

24. Kitagawa, T. et al. Observation of topologically protected bound states in a one dimensional photonic system. arXiv:quant-ph/1105.5334 (2011).

25. National Energy Research Supercomputing Center Annual Report. (2010).at $<$ http://www.nersc.gov>

26. Head-Gordon, M. \& Artacho, E. Chemistry on the computer. Physics Today 61, 58 (2008).

27. Sokolov, A.N. et al. From in silico to carbon to device: Computational discovery and experimental characterization of a high hole mobility organic crystal. In review (2011).

28. Aspuru-Guzik, A., Dutoi, A., Love, P. \& Head-Gordon, M. Simulated Quantum Computation of Molecular Energies. Science 309, 1704-1707 (2005).

29. Kassal, I., Whitfield, J.D., Perdomo-Ortiz, A., Yung, M.-H. \& Aspuru-Guzik, A. Simulating Chemistry Using Quantum Computers. Annual Review of Physical Chemistry 62, 185-207 (2011).

30. Schuch, N. \& Verstraete, F. Computational complexity of interacting electrons and fundamental limitations of density functional theory. Nat Phys 5, 732-735 (2009). 
31. Aaronson, S. \& Arkhipov, A. The Computational Complexity of Linear Optics. arXiv:quant-ph/1011.3245v1 (2010).

32. Whitfield, J.D., Biamonte, J. \& Aspuru-Guzik, A. Simulation of electronic structure Hamiltonians using quantum computers. Molecular Physics 109, 735750 (2011).

33. Abrams, D. \& Lloyd, S. Simulation of Many-Body Fermi Systems on a Universal Quantum Computer. Phys. Rev. Lett. 79, 2586-2586 (1997).

34. Abrams, D. \& Lloyd, S. Quantum Algorithm Providing Exponential Speed Increase for Finding Eigenvalues and Eigenvectors. Phys. Rev. Lett. 83, 51625162 (1999).

35. Aspuru-Guzik, A., Dutoi, A., Love, P. \& Head-Gordon, M. Simulated Quantum Computation of Molecular Energies. Science 309, 1704-1707 (2005).

36. Lanyon, B.P. et al. Simplifying quantum logic using higher-dimensional Hilbert spaces. Nat Phys 5, 134-140 (2009).

37. Reck, M., Zeilinger, A., Bernstein, H.J. \& Bertani, P. Experimental realization of any discrete unitary operator. Phys. Rev. Lett. 73, 58-61 (1994).

38. Politi, A., Cryan, M.J., Rarity, J.G., Yu, S. \& O’Brien, J.L. Silica-on-Silicon Waveguide Quantum Circuits. Science 320, 646-649 (2008).

39. Sansoni, L. et al. Polarization entangled state measurement on a chip. Phys. Rev. Lett. 105, 200503 (2010).

40. Owens, J.O. et al. Two-photon quantum walks in an elliptical direct-write waveguide array. New J. Phys. 13, 075003 (2011).

41. Perets, H.B. et al. Realization of Quantum Walks with Negligible Decoherence in Waveguide Lattices. Phys. Rev. Lett. 100, 170506 (2008).

42. Farhi, E. \& Gutmann, S. Quantum computation and decision trees. Phys. Rev. A 58, 915-928 (1998).

43. Whitfield, J.D., Rodríguez-Rosario, C.A. \& Aspuru-Guzik, A. Quantum stochastic walks: A generalization of classical random walks and quantum walks. Phys. Rev. A 81, 022323 (2010).

44. Broome, M.A. et al. Discrete Single-Photon Quantum Walks with Tunable Decoherence. Phys. Rev. Lett. 104, 153602 (2010).

45. Schreiber, A. et al. Photons Walking the Line: A Quantum Walk with Adjustable Coin Operations. Phys.Rev. Lett. 104, 050502 (2010). 
46. Schreiber, A. et al. Decoherence and Disorder in Quantum Walks: From Ballistic Spread to Localization. Phys. Rev. Lett. 106, 180403 (2011).

47. Matthews, J., Politi, A., Stefanov, A. \& O’Brien, J. Manipulation of multiphoton entanglement in waveguide quantum circuits. Nat Photon 3, 346-350 (2009).

48. Engel, G. et al. Evidence for wavelike energy transfer through quantum coherence in photosynthetic systems. Nature 446, 782-786 (2007).

49. Collini, E. et al. Coherently wired light-harvesting in photosynthetic marine algae at ambient temperature. Nature 463, 644-647 (2010).

50. Panitchayangkoon, G. et al. Long-lived quantum coherence in photosynthetic complexes at physiological temperature. Proceedings of the National Academy of Sciences 107, $12766-12770$ (2010).

51. Mohseni, M., Rebentrost, P., Lloyd, S. \& Aspuru-Guzik, A. EnvironmentAssisted Quantum Walks in Energy Transfer of Photosynthetic Complexes. $J$. Chem. Phys. 129, 179124016 (2008).

52. Rebentrost, P., Mohseni, M., Kassal, I., Lloyd, S. \& Aspuru-Guzik, A. Environment-assisted quantum transport. New J. Phys. 11, 033003 (2009).

53. Plenio, M.B. \& Huelga, S.F. Dephasing assisted transport: Quantum networks and biomolecules. New J. Phys. 10, (2008).

54. Whitfield, J.D., Rodríguez-Rosario, C.A. \& Aspuru-Guzik, A. Quantum stochastic walks: A generalization of classical random walks and quantum walks. Phys. Rev. A 81, 022323 (2010).

55. Caruso, F., Spagnolo, N., Vitelli, C., Sciarrino, F. \& Plenio, M.B. Simulation of noise-assisted transport via optical cavity networks. Phys. Rev. A 83, 013811 (2011).

56. Marshall, W. Antiferromagnetism. Proc. R. Soc. A232, 48 (1955).

57. Anderson, P.W. The Resonating Valence Bond State in $\mathrm{La}_{2} \mathrm{CuO}_{4}$ and Superconductivity. Science 235, 1196 -1198 (1987).

58. Coffman, V., Kundu, J. \& Wootters, W.K. Distributed entanglement. Phys. Rev. A 61, 052306 (2000).

59. Osborne, T.J. \& Verstraete, F. General Monogamy Inequality for Bipartite Qubit Entanglement. Phys. Rev. Lett. 96, 220503 (2006).

60. Mattle, K., Michler, M, Weinfurter, H., Zeilinger, A. \& Zukowski, M. NonClassical Statistics at Multiport Beam Splitters. Appl. Phys. B 60, 111-117 
61. Hong, C.K., Ou, Z.Y. \& Mandel, L. Measurement of subpicosecond time intervals between two photons by interference. Phys. Rev. Lett. 59, 2044-2046 (1987).

62. Braunstein, S.L. \& Mann, A. Measurement of the Bell operator and quantum teleportation. Phys. Rev. A 51, R1727-R1730 (1995).

63. Bouwmeester, D. et al. Experimental quantum teleportation. Nature 390, 575579 (1997).

64. Semião, F.L. \& Paternostro, M. Quantum circuits for spin and flavor degrees of freedom of quarks forming nucleons, Quant. Inf. Proc. doi:10.1007/s11128-0110232-3 (2011).

65. O’Brien, J.L., Furusawa, A. \& Vuckovic, J. Photonic quantum technologies. Nat Photon 3, 687-695 (2009).

66. Knill, E., Laflamme, R. \& Milburn, G.J. A scheme for efficient quantum computation with linear optics. Nature 409, 46-52 (2001).

67. Kok, P. et al. Linear optical quantum computing with photonic qubits. Rev. Mod. Phys. 79, 135-174 (2007).

68. Kwiat, P.G. et al. New High-Intensity Source of Polarization-Entangled Photon Pairs. Phys. Rev. Lett. 75, 4337-4341 (1995).

69. Rosfjord, K.M. et al. Nanowire single-photon detector with an integrated optical cavity and anti-reflection coating. Opt. Express 14, 527-534 (2006).

70. Divochiy, A. et al. Superconducting nanowire photon-number-resolving detector at telecommunication wavelengths. Nat Photon 2, 302-306 (2008).

71. Lita, A.E., Miller, A.J. \& Nam, S.W. Counting near-infrared single-photons with 95\% efficiency. Opt. Express 16, 3032-3040 (2008).

72. Hadfield, R.H. Single-photon detectors for optical quantum information applications. Nature Photon. 3, 696-705 (2009).

73. Tanner, M.G. et al. Enhanced telecom wavelength single-photon detection with NbTiN superconducting nanowires on oxidized silicon. Appl. Phys. Lett. 96, 221109 (2010).

74. Gerrits, T. et al. On-chip, photon-number-resolving, telecom-band detectors for scalable photonic information processing. arXiv:quant-ph/1107.5557 (2011).

75. Pernice, W. et al. High Speed Travelling Wave Single-Photon Detectors With Near-Unity Quantum Efficiency. arXiv:quant-ph/1108.5299 (2011). 
76. Ufimtsev, I.S., Luehr, N. \& Martinez, T.J. Charge Transfer and Polarization in Solvated Proteins from Ab Initio Molecular Dynamics. J. Phys. Chem. Lett. 2, 1789-1793 (2011).

77. Kassal, I., Jordan, S.P., Love, P.J., Mohseni, M. \& Aspuru-Guzik, A. Polynomial-time quantum algorithm for the simulation of chemical dynamics. Proceedings of the National Academy of Sciences 105, 18681-18686 (2008). 